\title{
Matching Raw GPS Measurements on a Navigable Map Without Computing a Global Position
}

\author{
Clément Fouque and Philippe Bonnifait
}

\begin{abstract}
Map-matching means determining the location of a mobile with respect to a road network description stored in a digital map. This problem is usually addressed using GPSlike fixes. Unfortunately, there are many situations in urban areas where few satellites are visible because of outages due to tall buildings. In this paper, map-matching is solved using raw GPS measurements (pseudoranges and Doppler measurements), avoiding the necessity to compute a global position. The problem is formalized in a general Bayesian framework in order to handle noise, which is able to perform multi-hypothesis map-matching when there is not enough information to make unambiguous decisions. This tightly-coupled GPS-Map fusion has to cope simultaneously with identifying the road and estimating the mobile's position on that road. A marginalized particle filter is proposed for solving this hybrid estimation problem efficiently. Real experimental results are reported to show that this approach can be initialized with fewer than four satellites. It is also able to track the location with two satellites only, once the road selection has been solved.
\end{abstract}

Key words: Map-Matching, Global Positioning Systems (GPS), Hybrid state estimation, particle filtering.

\section{INTRODUCTION}

Map-matching is a key element in driving assistance and is a feature of many Intelligent Transportation Systems (ITS) [22]. In a common map-matching approach [10], [23], [27], [7], a vehicle's global position needs to be obtained before its location with respect to the representation of the road network can be estimated. This pivotal issue for ITS has often been addressed using either topology or the geometry of the network [17], [28]. Fundamentally, it is a hybrid problem: a symbolic road identification needs to be combined with a metric positioning relative to the origin of the road. For instance, the authors of [14] include a prior estimation step to compute the pose of the vehicle using the fix of a GPS receiver. Then, this estimation is used to select the corresponding road from the map using a nearest-neighbor approach. Managing positioning and map uncertainties is of prime importance for map-matching. For this, several theoretical framework can be used. In [23], the road identification is performed using a Bayesian network. This selection can also be performed using Belief theory as proposed in [7] and in[1]. In [16], the map-matching approach makes use of a strategy that is formalized using Bayesian filtering. The main drawback of such approaches is the quality of the positioning solution, which is highly dependent on the availability and performance

Clément Fouque is with the Institut Pascal UMR CNRS 6602 and Philippe Bonnifait is with Heudiasyc UMR CNRS 7253, University of Technology of Compiegne (UTC), France, e-mail: philippe.bonnifait@hds.utc.fr. of the GPS positioning. In urban areas, GPS performance can often be poor because of signal outages or multi-path.

To overcome these limitations, an alternative approach consists in solving map-matching simultaneously with positioning using the raw measurements provided by a GPS receiver. In [8], a snapshot approach has been proposed to perform mapmatching using a coherency test with the GPS pseudoranges. Since this approach takes no account of topology, ambiguity and mismatching may occur in cases of parallel roads or at junctions. The author of [24] makes use of historic path data to solve this problem. The matching is then confirmed using the measurements likelihood. Furthermore, in [25] it has been shown that GPS corrections can be done using the map geometry. Such a tightly-coupled approach increases the map-matching precision that is usually degraded by offsets affecting the pseudoranges and the map.

A major concern with map-matching is frequent ambiguity at road junctions or when positioning accuracy is low with respect to the detailed geometry of the map. One technique for addressing this problem is to use multi-hypothesis tracking. The authors of [13] have proposed a Multi-Hypothesis Tracking (MHT) approach that uses road connectivity to merge the map information with GPS data. Hypotheses are attached to roads and new hypotheses are created at each new road junction. A similar approach was proposed by [18]. Constraint approaches can also be employed, as in [5], where an Interacting Multiple Model (IMM) is used to handle ambiguities at road junctions: When entering the vicinity of a road junction, a bank of map-constrained Extended Kalman Filters (EKF) are used. Similar approaches rely on constrained Particle Filters (PF) [12], [15]. Here, the evolution of the particles is constrained by the road geometry and, when a junction is reached, particles are allocated to connected roads according to existing knowledge of the topology of the network. Such an approach is also considered in [4]. In this paper, we also address the question of Multi-Hypothesis MapMatching (MHMM) which provides a means to avoid resetting the filter each time a mismatch occurs. Moreover, we will show that MHMM is also an efficient technique for initializing the method in challenging conditions with few GPS satellites.

Solving MHMM with raw GPS measurements consists in simultaneously identifying the roads and the corresponding locations of every hypothesis regarding these roads. It is a constraint positioning problem, since the space defined by the network geometry needs to be searched for every possible candidate. A Bayesian formalization provides an interesting framework for handling noise present in the measurements. Moreover, in such a framework, MHMM can be stated as 
a hybrid state estimation problem in the form of a Jump Markov System. In [4], this has been addressed in a looselycoupled way. We here extend this approach to a tightlycoupled PF using raw GPS measurements, i.e. Pseudoranges (PR) and Dopplers (also known as shifts of satellite frequency). Hypothesis estimation can thus be performed without any prior positioning stage, and every available GPS measurement can be used, even if there is not enough information to fix a global position, as can occur frequently in urban areas. An efficient factorization of MHMM can be done, in order to isolate the estimation of continuous components from the estimation of discrete ones. A marginalized particle filter can then be implemented to perform the estimation process with a limited number of particles.

The paper is organized as follows. In section II, the MHMM problem is stated in a generic Bayesian framework and the positioning estimation problem is described. Section IV presents an application of this general method to a specific problem: PR and Doppler GPS measurements are combined with a $3 \mathrm{D}$ road map to solve the map-matching problem. For an efficient implementation, an MPF for taking advantage of network topology is described. Finally, experimental results obtained in a situation with few visible satellites are reported in order to provide an indication of the solver performance as regards positioning quality and road identification in different scenarios.

\section{BAYESIAN MHMM}

This section provides a formalization of MHMM irrespective of both the type of sensor used and the method of representation of roads (which may take the form of polylines or clothoids, for instance). A general Bayesian framework is used to handle noise while being able to do MHMM when there is not enough information to make unambiguous decisions.

\section{A. Map representation}

In this work, a navigable road map is assumed to describe the road network using one carriageway per driving direction. So, a normal two-way road is represented by two carriageways having the same geometry but opposite directions. Moreover, the connections (also called nodes) between the carriageways are known and stored in the digital map.

Since a navigable road map can represent a large amount of data, a limited area of the map has to be considered. Below this will be referred to as the "road cache". In [3], a method is described for efficiently handling a map managed by a Geographical Information System (GIS) with two overlapping road caches. In the following paragraphs, all the mathematical developments are done for one road cache that is assumed to be sufficiently large for the method to converge. In practice, managing several road caches becomes a problem only where some hypotheses need to be discarded.

\section{B. State-space model}

A map-matching problem can be seen as a hybrid state estimation problem. Each carriageway is identified by a unique identification (ID), denoted $\mathcal{I}$, that is discrete. This description may be extended to address the lane-matching problem [27] where a road having several lanes is modeled using as many polylines as lanes.

The location on a carriageway is described by the curvilinear abscissa, denoted $l$, which is a continuous parameter. Some additional parameters may be needed to solve the state observation problem, as we shall see later on. So, let $x$ (one of whose components is $l$ ) denote the vector that contains all the continuous components. We consider a hybrid state vector $s_{k}$ where the subscript is used to indicate a sample:

$$
s_{k}=\left[\begin{array}{ll}
\mathcal{I}_{k} & x_{k}
\end{array}\right]^{T} \in \mathbb{N} \times \mathbb{R}^{n}
$$

In [6], a similar problem is addressed for real-time diagnosis, where unknown discrete states are used to describe normal operation and faulty conditions. We adopt here the same notation to formalize the MHMM problem using the following stochastic model:

$$
\left\{\begin{array}{l}
\mathcal{I}_{k+1} \sim P\left(\mathcal{I}_{k+1}=\mathcal{I} \mid \mathcal{I}_{k}, x_{k}, y_{k}\right) \\
x_{k+1}=f\left(\mathcal{I}_{k}, x_{k}\right)+\alpha_{k} \\
y_{k}=g\left(\mathcal{I}_{k}, x_{k}\right)+\beta_{k}
\end{array}\right.
$$

where $y_{k}$ is the observation vector at step $k$ using the sensors, $\alpha_{k}$ the process error and $\beta_{k}$ the observation error. $f($.$) is the process model describing the evolution of the$ continuous components and $g($.$) the observation model. These$ models depend on the carriageway ID because of the map constraint. Finally, $P($.$) is a transition kernel depicting the$ evolution of the probability of the IDs $\mathcal{I}$ contained in the map. Below we assume a first order Markov chain, depending on the network topology. Thus, (2) is a Jump Markov System.

\section{Bayesian sequential estimation}

We recall here the classical Bayesian theory that allows the state of a system perturbed by noises and errors like in Eq. (2) to be estimated. The problem consists in estimating sequentially the Probability Density Function (PDF) $p\left(s_{k} \mid y_{1: k}\right)$ using all the available measurements $y_{1: k}$ from the start time to the current time. This can be done using an estimation/prediction mechanism.

Using Bayes factorization, the posterior density can be rewritten as:

$$
p\left(s_{k} \mid y_{1: k}\right)=p\left(y_{k} \mid s_{k}\right) \cdot \frac{p\left(s_{k} \mid y_{1: k-1}\right)}{p\left(y_{k} \mid y_{1: k}\right)}
$$

where $p\left(y_{k} \mid s_{k}\right)$ is the observation likelihood according to the observation model of (2) and $p\left(y_{k} \mid y_{1: k}\right)$ a normalization factor given by:

$$
p\left(y_{k} \mid y_{1: k-1}\right)=\int p\left(y_{k} \mid s_{k}\right) \cdot p\left(s_{k} \mid y_{1: k-1}\right) \cdot d s_{k}
$$

This step needs $p\left(s_{k} \mid y_{1: k-1}\right)$, the prior PDF of $s_{k}$ (also called prediction) which can be estimated using the process model and the posterior at step $k-1$ : 


$$
p\left(s_{k} \mid y_{1: k-1}\right)=\int p\left(s_{k} \mid s_{k-1}\right) p\left(s_{k-1} \mid y_{1: k-1}\right) d s_{k-1}
$$

Equations (3) and (5) correspond to the estimation and prediction stages of the Bayesian MHMM.

\section{Solutions to Bayesian MHMM}

In a limited number of cases, Bayesian filtering has an analytical solution. For instance, if the equations are linear, and if the noises are Gaussian, centered and additive, then the solution is the Kalman filter. To the best of our knowledge, MHMM has no analytical solution, mainly because of the road network that gives rise to bifurcations in the evolution model. Bifurcations are particularly difficult nonlinearities.

Monte Carlo methods provide alternative numerical methods for solving nonlinear Bayesian problems [2]. Many numerical approximation schemes have been studied in the literature, particle filtering being the most popular.

As shown in [4], MHMM can be solved using particle filtering by sampling directly all the dimensions of the state. Nevertheless, factoring the PDF can significantly reduce the computational complexity of the problem.

Let us now explicitly consider the discrete and continuous components of $s_{k}=\left[\begin{array}{ll}\mathcal{I}_{k} & x_{k}\end{array}\right]^{T}$. The joint posterior $p\left(x_{k}, \mathcal{I}_{k} \mid y_{1: k}\right)$ can be factored according to Bayes' rule:

$$
p\left(x_{k}, \mathcal{I}_{k} \mid y_{1: k}\right)=p\left(x_{k} \mid \mathcal{I}_{k}, y_{1: k}\right) \cdot p\left(\mathcal{I}_{k} \mid y_{1: k}\right)
$$

This factoring separates the problem of carriageway identification, described by $p\left(\mathcal{I}_{k} \mid y_{1: k}\right)$, from the problem of localizing the mobile on the carriageway, depicted by $p\left(x_{k} \mid \mathcal{I}_{k}, y_{1: k}\right)$. Interestingly, the physical interpretation corresponds to a very common map-matching strategy, namely find the road and then find where you are on the road.

This kind of factoring is often called "RaoBlackwellization" [6], [21]. For instance in [11], it is used to accelerate a PF to solve the problem of Simultaneous Localization And Mapping (SLAM) and in [12] the factoring helps reducing the number of particles for tracking a target. Below we shall use the term MPF like "Marginalized PF".

In Eq. (6), $p\left(x_{k} \mid \mathcal{I}_{k}, y_{1: k}\right)$ can be estimated using Kalman filtering since the continuous components are isolated from the carriageways. $p\left(\mathcal{I}_{k} \mid y_{1: k}\right)$ can be sampled by a sequential Monte Carlo using importance sampling with particles [19]. In other words, hypotheses are attached to every candidate carriageway and several EKFs are used to track the continuous components along it. New candidate carriageways are randomly chosen at junctions using the connection information stored in the map.

\section{E. Estimation of map-matched locations}

From (6) we may obtain the most likely positioning solution in the entire road cache.

This strategy is not always the best, especially if the identification of the carriageway is ambiguous. In this case, it may be preferable to perform an estimation on a per-carriageway basis. For a given carriageway $\mathcal{I}$, the positioning hypothesis $M_{k}^{\mathcal{I}}$ is characterized by its posterior and its probability with respect to the others:

$$
M_{k}^{\mathcal{I}}=\left\{\begin{array}{l}
p\left(x_{k} \mid \mathcal{I}_{k}=\mathcal{I}, y_{1: k}\right) \\
\Omega_{k}^{\mathcal{I}}=P\left(\mathcal{I}_{k}=\mathcal{I} \mid y_{1: k}\right)
\end{array} \quad \text { with } \sum_{i=1}^{N_{\mathcal{I}}} \Omega_{k}^{\mathcal{I}}=1\right.
$$

If several hypotheses have significant scores $\Omega_{k}^{\mathcal{I}}$, MHMM has multiple solutions.

\section{MARginALIZED PF DEVELOPMENT}

The Bayesian MHMM problem is solved using an MPF to reduce the complexity and improve the robustness. Next, the carriageway identification is performed by a bootstrap PF.

\section{A. Continuous component tracking}

As EKFs estimate the continuous components, errors are assumed to be zero mean and Gaussian. Thus, process and observation errors are modeled by Gaussian white noises: $\alpha_{k} \sim \mathcal{N}\left(0, Q_{\alpha}\right)$ and $\beta_{k} \sim \mathcal{N}\left(0, Q_{\beta}\right)$. The candidate positions are approximated by Gaussian densities along the carriageways. Their mean $\mu_{k \mid k}$ and covariance $\Sigma_{k \mid k}$ define the probability density function:

$$
p\left(x_{k \mid k} \mid \mathcal{I}_{k}, y_{1: k}\right) \simeq \mathcal{N}\left(x_{k \mid k} ; \mu_{k \mid k}, \Sigma_{k \mid k}\right)
$$

where $\mathcal{N}\left(x_{k} ; \mu_{k}, \Sigma_{k}\right)$ denotes Gaussian distribution of random variable $x$ given by its mean $\mu_{k}$ and covariance matrix $\Sigma_{k}$.

EKF correction estimates the mean and covariance $\mu_{k \mid k}$ and $\Sigma_{k \mid k}$ through the use of the optimal Kalman matrix $K$ (see [26] for a demonstration):

$$
\left\{\begin{array}{l}
\Gamma_{k}^{\mathcal{I}}=G_{k}^{\mathcal{I}} \cdot \Sigma_{k \mid k-1} \cdot\left(G_{k}^{\mathcal{I}}\right)^{T}+Q_{\beta} \\
K=\Sigma_{k \mid k-1} \cdot\left(G_{k}^{\mathcal{I}}\right)^{T} \cdot\left(\Gamma_{k}^{\mathcal{I}}\right)^{-1} \\
\mu_{k \mid k}=\mu_{k \mid k-1}+K\left(y_{k}-g\left(\mathcal{I}_{k}, \mu_{k \mid k-1}\right)\right) \\
\Sigma_{k \mid k}=\left(\mathrm{I}-K \cdot G_{k}^{\mathcal{I}}\right) \cdot \Sigma_{k \mid k-1}
\end{array}\right.
$$

where $G_{k}^{\mathcal{I}}=\frac{\partial g\left(\mathcal{I}_{k}, x_{k}\right)}{\partial x}$ is the Jacobian matrix of the constrained observation model evaluated at the prediction stage.

The prediction stage is given by:

$$
\left\{\begin{array}{l}
\mu_{k+1 \mid k}=f\left(\mathcal{I}_{k}, \mu_{k \mid k}\right) \\
\Sigma_{k+1 \mid k}=F_{k}^{\mathcal{I}} \cdot \Sigma_{k \mid k} \cdot\left(F_{k}^{\mathcal{I}}\right)^{T}+Q_{\alpha}
\end{array}\right.
$$

where $F_{k}^{\mathcal{I}}=\frac{\partial f\left(\mathcal{I}_{k}, x_{k}\right)}{\partial x}$ is the Jacobian matrix of the constrained process model evaluated according to $\mu_{k \mid k}$. $\mathcal{I}_{k}$ is the carriageway ID corresponding to the location of the considered hypothesis. One might remark here that the hypothesis may go past the end of the carriageway during the prediction stage. In such a case, the length of the previous carriageway is subtracted from the curvilinear abscissa. In theory, a hypothesis may skip several roads if the displacement of the vehicle is longer than the following carriageway. Additionally, the process model must be piecewise differentiable to allow the Jacobian matrix $F_{k}^{\mathcal{I}}$ to be computed. This is the case in the following development. 


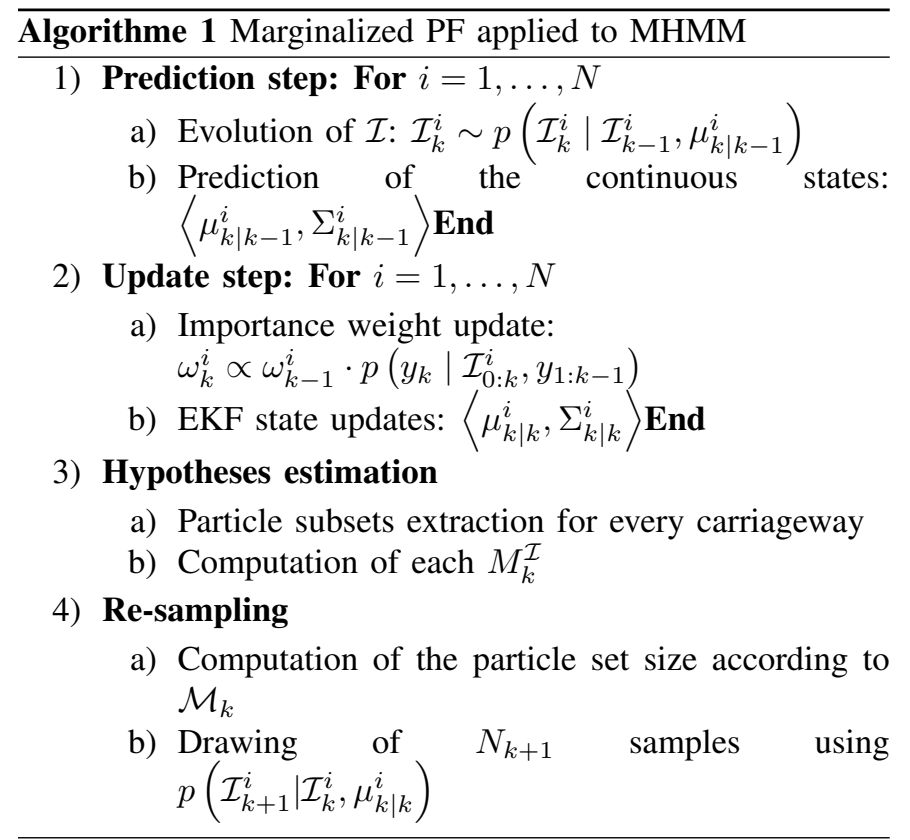

\section{B. Sampling of the IDs}

For this discrete part of the problem, we perform importance sampling.

Let us consider a set of $N$ weighted samples of $p\left(\mathcal{I}_{k} \mid y_{1: k}\right)$ :

$$
\chi_{k}=\left\{\mathcal{I}_{k}^{i}, \omega_{k}^{i}\right\}_{i=1: N}
$$

where $\omega_{k}^{i}$ denotes the weight. Therefore, the density describing the road identification problem is approximated by:

$$
p\left(\mathcal{I}_{k} \mid y_{1: k}\right)=\sum_{i=1}^{N} \omega_{k}^{i} \cdot \delta_{\mathcal{I}_{k}}\left(\mathcal{I}^{i}\right)
$$

where $\delta_{\mathcal{I}_{k}}\left(\mathcal{I}^{i}\right)$ is the Dirac delta function.

To estimate the weight of every particle, standard bootstrap [19] is applied for the recursion:

$$
\left\{\begin{array}{l}
\gamma_{k}^{i}=p\left(y_{k} \mid \mathcal{I}_{0: k}^{i}, y_{1: k-1}\right) \\
\omega_{k}^{i}=\omega_{k-1}^{i} \cdot \gamma_{k}^{i}
\end{array}\right.
$$

The measurement likelihood $\gamma_{k}^{i}$ of the current measurement $y_{k}$ is quantified using a Normal law, assuming that the measurement errors are Gaussian. It is computed according to the constraint predicted measurement $\widehat{y}_{k \mid k-1}^{i}$ of the EKF [21]:

$$
\gamma_{k}^{i}=\mathcal{N}\left(y_{k} ; \widehat{y}_{k \mid k-1}, \Gamma_{k}^{\mathcal{I}}\right)
$$

where $\Gamma_{k}^{\mathcal{I}}$ is the covariance of the innovation (see Eq. 9).

Then, weights are normalized such that their sum equals one.

\section{Algorithm}

Algorithm 1 describes the realization of the MPF. To avoid particle set degeneracy, a classical re-sampling strategy is used [19]. We have also chosen an adaptive approach, as proposed by the authors of [9]. The size of the particle set is then adjusted according to the number of likely hypotheses. When approaching a junction for instance, $N$ is automatically increased. $N$ is minimal when there is only one carriageway to track.

A positioning hypothesis (7) is obtained from the subset $\chi_{k}^{\mathcal{I}}$ of the particles moving along the given carriageway $\mathcal{I}$ through a Gaussian mixture:

$$
M_{k}^{\mathcal{I}}=\left\{\begin{array}{l}
\sum_{i=1}^{N_{k}^{\mathcal{I}}} \omega_{k}^{i} \cdot \mathcal{N}\left(x_{k} ; \mu_{k \mid k}^{i}, \Sigma_{k \mid k}^{i}\right) \\
\Omega_{k}^{\mathcal{I}}=\sum_{i=1}^{N_{k}^{\mathcal{I}}} \omega_{k}^{i}
\end{array}\right.
$$

where $N_{k}^{\mathcal{I}}$ is the dimension of this particle subset. Thus, the hypothesis mean state is given by:

$$
\mu_{k}^{\mathcal{I}}=\frac{1}{\Omega_{k}^{\mathcal{I}}} \cdot \sum_{i=1}^{N_{k}^{r}} \omega_{k}^{i} \cdot \mu_{k \mid k}^{i}
$$

and its covariance is obtained by:

$$
\Sigma_{k}^{\mathcal{I}}=\frac{1}{\Omega_{k}^{\mathcal{I}}} \cdot \sum_{i=1}^{N_{k}^{\mathcal{I}}} \omega_{k}^{i} \cdot\left(\Sigma_{k \mid k}^{i}+\left(\mu_{k \mid k}^{i}-\mu_{k}^{\mathcal{I}}\right)^{2}\right)
$$

The estimated covariance of the hypothesis state is also an interesting feature of this approach. It means that a confidence interval can be estimated for every map-matched solution.

\section{SOlVING THE MHMM USING RAW GPS AND POLYLINES}

In this section, we show how to solve the Bayesian MHMM when using raw GPS measurements and when the carriageways are described by polylines. A measurement of vehicle speed is also used.

Since we use raw GPS measurements to compute the Bayesian inference, additional continuous parameters are needed, so:

$$
x_{k}=\left[\begin{array}{llll}
l_{k} & v_{k} & d_{k} & \dot{d}_{k}
\end{array}\right]
$$

where $v_{k}$ is the speed along the carriageway. $d_{k}$ and $\dot{d}_{k}$ represent the clock offset and the clock drift of the receiver, respectively multiplied by the speed of light in vacuum.

\section{A. Evolution of the carriageway IDs}

The kernel transition for the carriageway IDs $\mathcal{I}$ is given by a topological approach: if a hypothesis reaches the end of its carriageway, the transition depends on the connected carriageways $\mathcal{C}^{\mathcal{I}}$. By assuming an equiprobable transition, the sampling can be done as follows:

$$
\left\{\begin{array}{l}
P\left(\mathcal{I}_{k+1}=\mathcal{I}_{k} \mid \mathcal{I}_{k}, l_{k}<L^{\mathcal{I}_{k}}\right)=1 \\
P\left(\mathcal{I}_{k+1}=\mathcal{J} \mid \mathcal{I}_{k}, l_{k} \geq L^{\mathcal{I}_{k}}\right)=\frac{1}{\operatorname{dim} \mathcal{C}^{\mathcal{I}}} \quad \forall \mathcal{J} \in \mathcal{C}^{\mathcal{I}}
\end{array}\right.
$$

where $L^{\mathcal{I}_{k}}$ is the total length of carriageway $\mathcal{I}_{k}$. 


\section{B. Evolution of the continuous components}

Next, the process model for the continuous components is introduced.

1) Process Model: As constrained hypotheses are used, the process model $x_{k+1}=f\left(\mathcal{I}_{k}, x_{k}\right)$ reduces to a conditionally linear model. When moving along a carriageway, the process model is equivalent to an integrator of the hypothesis speed and clock drift. So, the process model is given by:

$$
x_{k+1}= \begin{cases}A \cdot x_{k}+B \cdot u_{k}+C \cdot L_{k}^{\mathcal{I}} & \text { if } \mathcal{I}_{k+1} \neq \mathcal{I}_{k} \\ A \cdot x_{k}+B \cdot u_{k} & \text { else }\end{cases}
$$

With a constant sampling period $T_{e}$, the process matrices $A$ and $B$ are invariant and given by:

$$
A=\left[\begin{array}{cccc}
1 & T_{e} & 0 & 0 \\
0 & 1 & 0 & 0 \\
0 & 0 & 1 & T_{e} \\
0 & 0 & 0 & 1
\end{array}\right] \quad B=\left[\begin{array}{l}
0 \\
1 \\
0 \\
0
\end{array}\right]
$$

Matrix $C$ is useful to reset the curvilinear abscissa when switching from one road to another. It is given by:

$$
C=\left[\begin{array}{llll}
-1 & 0 & 0 & 0
\end{array}\right]^{T}
$$

From Eq. (20) it can be seen that the evolution model is nonlinear owing to discontinuities at road junctions. This induces a reinitialization of the curvilinear abscissa. Moreover, these discontinuities can be considered as deterministic changes that only affect the curvilinear abscissa since, in the expression of the PDF $p\left(x_{k} \mid \mathcal{I}_{k}, y_{1: k}\right)$, the IDs are known. As a result, the variance of the prediction error is not affected by the discontinuities and can be computed using a linear expression. This is a nice characteristic of this formalization.

2) Random input: To allow changes in hypothesis velocity, a model noise is added in the form of a random input of the process model. In order to quantify the variance of this shaping parameter, one needs to consider the confidence in the model with respect to the road geometry. Indeed, if the vehicle is moving along a straight road, there is a strong similarity between the distance covered along the polyline and the effective motion. Thus, one can have confidence in the map. On the other hand, when the vehicle undergoes a sharp turn, the similarity decreases because often maps are roughly sampled. An efficient way of dealing with this kind of situation is to make the particle explore the network in a random fashion, in which case the input $u_{k}$ that is applied to every particle, is drawn from a Gaussian white noise:

$$
u_{k} \propto \mathcal{N}\left(0, Q_{\zeta}\right)
$$

where the variance $Q_{\zeta}$ describes the confidence in the constant heading model. It is defined according to a measured yaw rate of the vehicle, denoted $\dot{\psi}$, obtained using a low-cost yaw-rate gyrometer. We propose using the following Gaussian model:

$$
Q_{\zeta}=\sigma_{\zeta}^{2} \cdot\left[1-\exp \left(-\frac{\dot{\psi}^{2}}{2 \sigma_{\dot{\psi}}^{2}}\right)\right]
$$

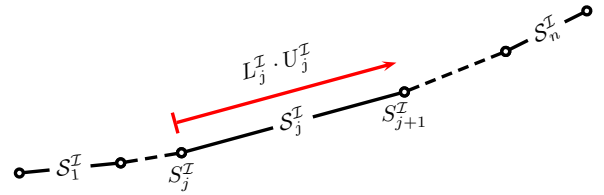

Figure 1: Geometrical description of a carriageway.

where $\sigma_{\dot{\psi}}$ is the standard deviation of the yaw-rate measurement and $\sigma_{\zeta}$ the maximum standard deviation of the model noise. This can be seen as a tuning parameter of the method. Thanks to the use of this mechanism, the confidence in the map geometry is introduced into the estimation process. We have noticed that it enhances the filter behavior, particularly when the spatial-sampling of the map is low and at junctions.

\section{Constrained observation models}

The main idea here is to use the raw GPS measurements, PR and Dopplers, and the speed of the vehicle to estimate the likelihood of every hypothesis. This section presents the tightly-coupled observation models.

1) Extraction of the geometrical parameters: Let us consider a polyline composed of $n+1$ segments. Each segment is defined by its origin $S_{i}^{\mathcal{I}}$, its length $L_{i}^{\mathcal{I}}$ and its orientation in space $U_{i}^{\mathcal{I}}$ (see Figure (1)). The last segment is set with $L_{n+1}^{\mathcal{I}}=0$. Here, we use 3D maps: $U_{i}^{\mathcal{I}}$ and $S_{i}^{\mathcal{I}}$ are 3D vectors.

To find the global position $X_{k}^{\mathcal{I}}$, we first need to extract the segment indexed $j$ such that:

$$
\sum_{i=1}^{j} L_{i}^{\mathcal{I}}<l<\sum_{i=1}^{j+1} L_{i}^{\mathcal{I}}
$$

Thus, we can express the position in the global frame in terms of the geometry of carriageway $\mathcal{I}$ :

$$
X_{k}^{\mathcal{I}}=S_{j}^{\mathcal{I}}+\left(l_{k}-\sum_{i=1}^{j-1} L_{i}^{\mathcal{I}}\right) \cdot U_{j}^{\mathcal{I}}
$$

Identically, the motion is also constrained: the vehicle direction is assumed to be collinear to the direction of carriageway $\mathcal{I}$ :

$$
V_{k}^{\mathcal{I}}=v_{k} \cdot U_{j}^{\mathcal{I}}
$$

The constraint on the vehicle motion means that vehicle directions can be used for estimating the likelihood of a hypothesis. Without this, the estimation relies on position only, which may lead to mismatches if map offsets are large.

2) Map-constrained models: Constrained position Eq.(26) - and velocity -Eq.(27)- are introduced in the standard observation models [20].

For a given satellite, the pseudorange is given by the geometrical range biased by the receiver clock offset $d_{k}$. Introducing the constrained position (26) in this model provides the constrained observation model for one satellite: 


$$
\rho_{k}^{\mathcal{I}}=\left\|S_{j}^{\mathcal{I}}+\left(l_{k}-\sum_{i=1}^{j-1} L_{i}^{\mathcal{I}}\right) \cdot U_{j}^{\mathcal{I}}-X_{k}^{s}\right\|+d_{k}
$$

where $X_{k}^{s}$ is the satellite position reconstructed from the broadcast ephemeris.

The same approach is used for the Doppler measurement. The expression of the constrained velocity (27) is therefore incorporated into the standard model. The Doppler measurement is given by a dot product (denoted $\bullet$ ):

$$
\dot{\rho}_{k}^{\mathcal{I}}=\left(v_{k} \cdot U_{j}^{\mathcal{I}}-V_{k}^{s}\right) \bullet u_{\operatorname{los}}+\dot{d}_{k}
$$

where $V_{k}^{s}$ is the satellite velocity obtained from the broadcast ephemeris [29], $u_{\operatorname{los}}$ is the line-of-sight vector between the receiver and the satellite, and $\dot{d}_{k}$ the clock drift of the receiver.

Since the position is constrained, $u_{\text {los }}$ depends also on the map geometry:

$$
u_{\mathrm{los}}=\frac{S_{j}^{\mathcal{I}}+\left(l_{k}-\sum_{i=1}^{j-1} L_{i}^{\mathcal{I}}\right) \cdot U_{j}^{\mathcal{I}}-X_{k}^{s}}{\left\|S_{j}^{\mathcal{I}}+\left(l_{k}-\sum_{i=1}^{j-1} L_{i}^{\mathcal{I}}\right) \cdot U_{j}^{\mathcal{I}}-X_{k}^{s}\right\|}
$$

Finally, the measurement likelihood $p\left(y_{k} \mid \mathcal{I}_{0: k}^{i}, y_{1: k-1}\right)$ is quantified according to the GPS raw measurements and the vehicle longitudinal speed, denoted $y_{v, k}$, provided by the vehicle. The measurement errors are assumed to be Gaussian for both sensors. Moreover, we assume that GPS measurements and the speed are not correlated, which is a reasonable assumption given that two independent sources of information are used. Using the same notation as in Eq. (14), the likelihood is given by:

$$
\begin{gathered}
\gamma_{k}=p\left(y_{k} \mid \mathcal{I}_{0: k}^{i}, y_{1: k-1}\right) \\
\gamma_{k}=\mathcal{N}\left(y_{v, k} ; \widehat{y}_{v, k}^{i}, \Gamma_{v, k}^{i}\right) \cdot \mathcal{N}\left(y_{G P S, k} ; \widehat{y}_{G P S, k}^{i}, \Gamma_{G P S, k}^{i}\right)
\end{gathered}
$$

where $y_{v, k}$ is the measured speed:

$$
y_{v, k}=\left[\begin{array}{llll}
0 & 1 & 0 & 0
\end{array}\right] \cdot x_{k}
$$

and $y_{G P S, k}$ the concatenation of all the available raw GPS measurements (two measures for each satellite in view). With $p$ visible satellites:

$$
y_{G P S, k}=\left[\begin{array}{llllll}
\rho_{1, k}^{\mathcal{I}} & \cdots & \rho_{p, k}^{\mathcal{I}} & \dot{\rho}_{1, k}^{\mathcal{I}} & \cdots & \dot{\rho}_{p, k}^{\mathcal{I}}
\end{array}\right]^{T}
$$

In order to implement the EKF, the Jacobian matrix of the observation models needs to be computed. The most important parameters are given in the appendix.

\section{Initialization of the particle set}

For a positioning system, initialization is a critical issue particularly in dense urban areas. Standard strategies require a GPS fix to initialize the positioning filter. In such conditions, at least 4 satellites are necessary before performing the tracking.

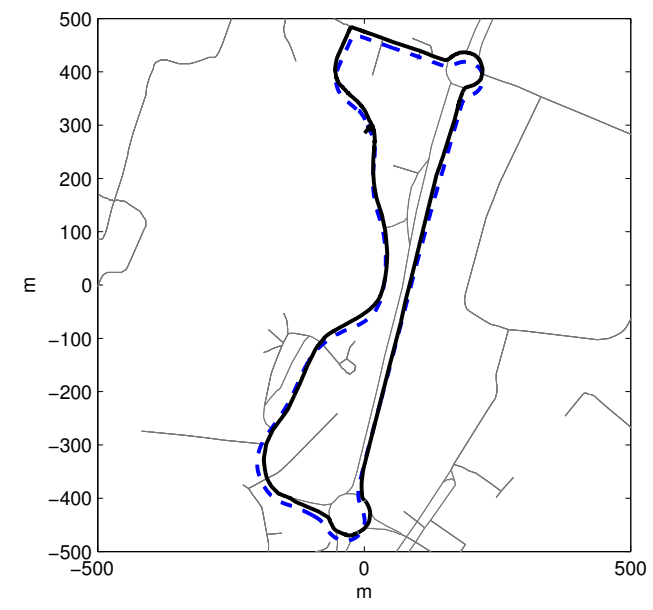

Figure 2: Trajectory -dashed-vs. map-matched trajectory -plain- in an ENU frame centered on the map.

The MHMM method proposed here is able to do the initialization by itself, provided that an appropriate particle set is used. For a correct convergence, the initialization process relies on two main assumptions. First, the current location of the vehicle has to be located inside the road cache. Secondly, the receiver clock dynamics (which are also unknown) have to be correctly sampled. For many GPS receivers, it is possible to know the clock offset boundaries and the magnitude of the clock drift. For the GPS receiver used in the experiments, it is also possible to select these values. Knowing these bounds, a particle set may be drawn such that the entire state-space is adequately covered.

In practice, the particle set can be initialized according to the following algorithm. Each carriageway in the road cache is uniformly sampled to provide a set of initial locations. For every candidate location, the receiver clock parameters are sampled according to the boundaries. Additionally, the velocity of every candidate is set according to the measured speed value. This approach solves the problem of tracking initialization with little prior knowledge of the vehicle location.

\section{EXPERIMENTAL RESULTS}

In this section, several experimental results are reported to show the performance of the MHMM approach. First, the experimental setup is summarized. Next, the benefit to be gained by using Doppler measurements when crossing a road junction is investigated. Map-matching performance is then evaluated in both open-sky and limited satellite visibility conditions. Finally, the initialization process is studied for a moving and a stationary vehicle.

\section{A. Experimental setup}

The proposed algorithm uses raw GPS measurements, a measure of the speed, and a measure of the yaw rate of the vehicle. Tests were carried out with our experimental car in the vicinity of Compiègne. The GPS measurements were provided by a Septentrio PolaRxze outputting raw data 


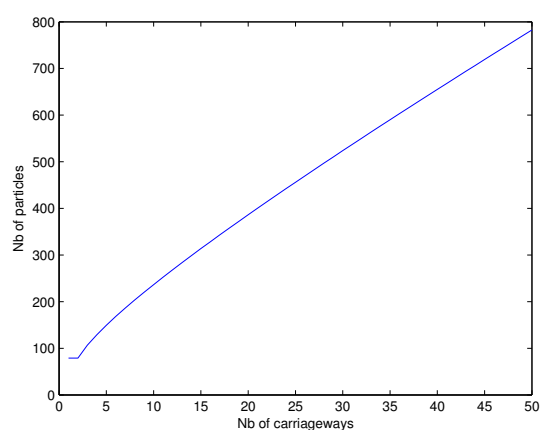

Figure 3: Dimension of the particle set according to the number of likely carriageways.

at $10 \mathrm{~Hz}$. Simultaneously, the speed and the gyro were logged through a gateway of the experimental vehicle. Data was post-processed using an accurate time-synchronization of the measurements.

A road cache of $750 \mathrm{~m}$ radius was extracted from a commercial TeleAtlas map. A working frame was set as an EastNorth-Up (ENU) frame centered on the map. Figure 2 shows the true trajectory -dashed-versus the map-matched trajectory -bold - and the road cache -light gray-. The TeleAtlas map used has an offset of roughly 15 meters in Compiègne.

Below, we compare the results of the road selection to a manually-performed map-matching using a method similar to the one presented in [4] for map-matching evaluation. The map-matching position is estimated using the curvilinear abscissa along the polylines see Eq.(26), and it is compared with the position computed by the PolaRxze.

\section{B. Tuning of the filter parameters}

The variance of the model noise $\sigma_{\zeta}$ is considered as a tuning parameter corresponding to the quality of the map. In these experiments, the navigable map has large biases and a low spatial sampling. Thus, we set a large variance for the model noise: $\sigma_{\zeta}=10 \mathrm{~m} . \mathrm{s}^{-1}$ and the variance of the yawrate measurement is set to $\sigma_{\dot{\psi}}=0.05 \mathrm{rad} . \mathrm{s}^{-1}$. As the model noise propagates through the process model, the model noise $Q_{\alpha}$ is set to zero for the velocity and the curvilinear abscissa. Conversely, a noise model $Q_{\alpha}$ is added to allow the variation of the receiver clock drift. It is set to $3 m . s^{-1}$.

In order to quantify the standard deviation of the PRs and Doppler measurements, we used broadcast EGNOS information. EGNOS is the European augmentation system similar to WAAS. Corrections (mainly ionospheric and tropospheric) were also applied individually to each PR using fast and long term corrections.

Figure 3 shows the number of particles used versus the number of carriageways. It will be remarked that the use of a MPF allows a limited number of particles to be used. Moreover, there is no exponential growth of this figure when a number of roads need to be tracked.

\section{Benefits of the Doppler measurements}

To illustrate the benefits of Dopplers for road identification, the crossing of a single road junction is considered in Figure 4.

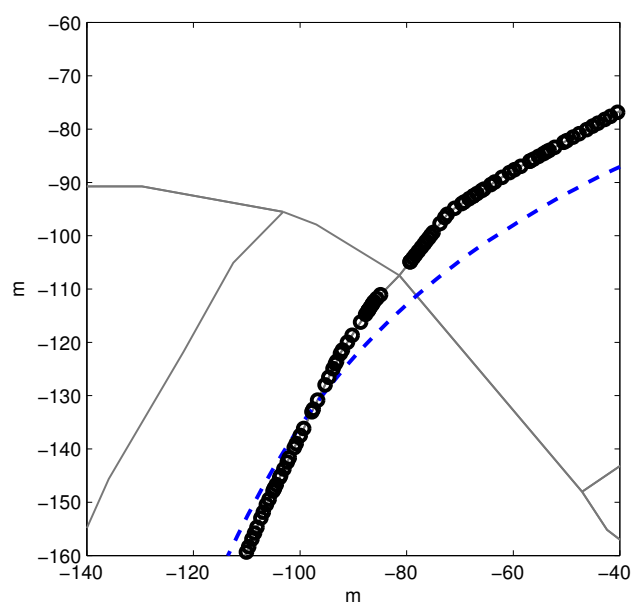

(a) With Dopplers.

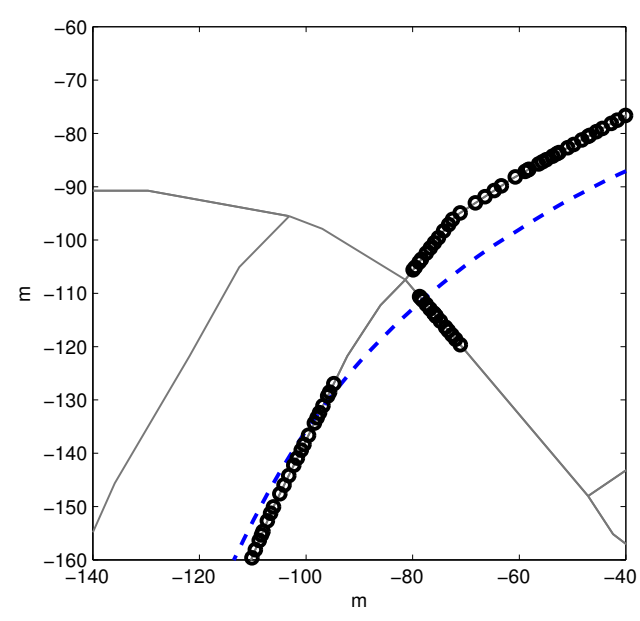

(b) Without Dopplers.

Figure 4: Vehicle path-dashed-versus map-matched position of the most likely hypothesis $-d o t$.

Two cases are considered: with PRs and Dopplers -4aand without Dopplers $-4 \mathrm{~b}$. Additionally, Figure 5 shows the variation of the hypothesis scores with respect to time. The scores of the correct carriageways are highlighted with bold lines. The maximum number of candidate carriageways is equal to five, since all the roads are two-directional (a hypothesis that reaches the end of the current carriageway can come back in the opposite direction).

Without Dopplers, road identification relies exclusively on the particles' positions through the constrained PR model of Eq.(28). Thus, several wrong selections are made at the junction, given that the map has an offset. When using the Dopplers, the road is correctly identified since identification makes use of the heading information provided by the Dopplers and expressed in Eq.(29). This phenomenon is also clearly illustrated by the way the hypothesis scores evolve. Without Dopplers, an ambiguity remains for 2.5 seconds (instead of less than 1 second with Dopplers), and the wrong hypothesis score can be significantly high. This is 

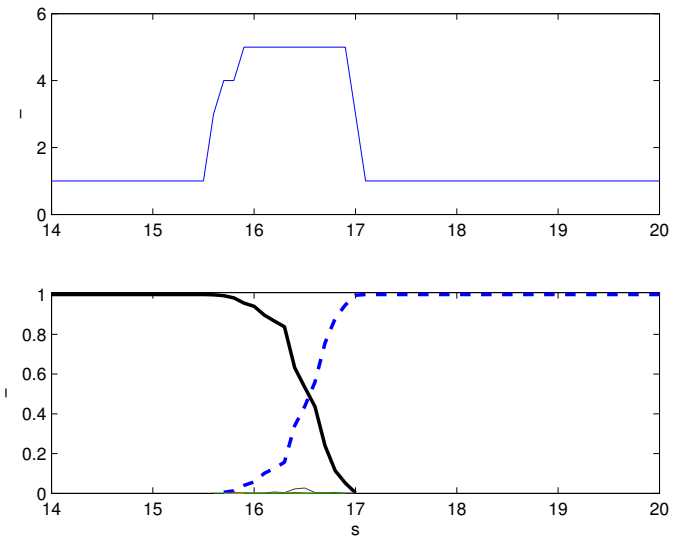

(a) With Dopplers
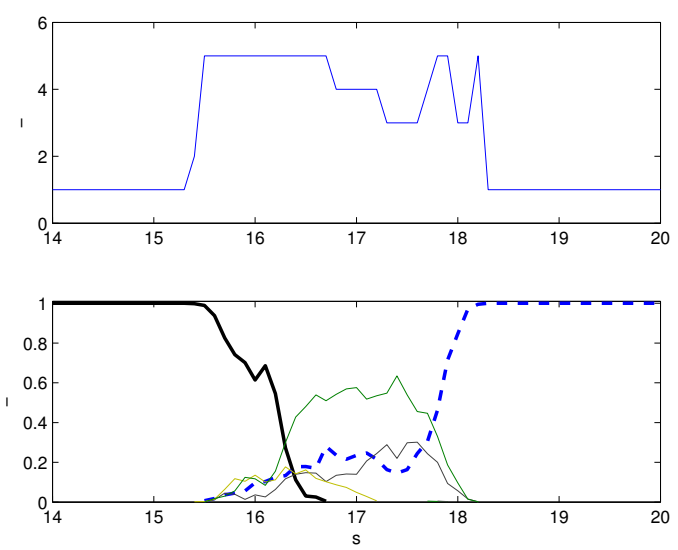

(b) Without Dopplers

Figure 5: Number of hypotheses -upper curve- and hypotheses relative likelihoods -lower curve. The correct carriageways are highlighted -bold lines.

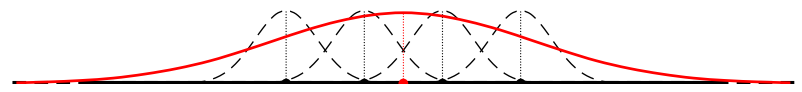

(a) Along a carriageway

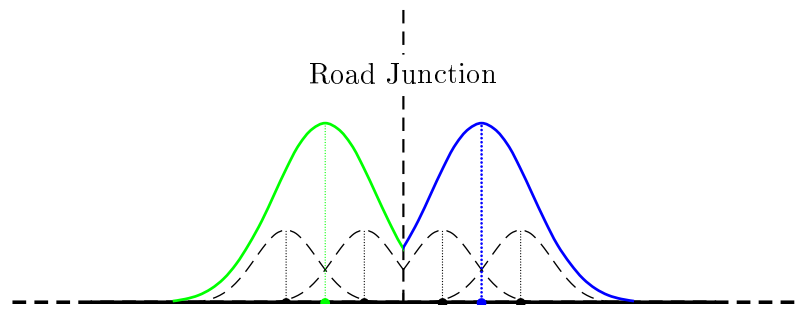

(b) At a road junction

Figure 6: Gaussian mixtures for two particle sets.

why a wrong matching occurs while crossing the junction in Figure 4b. So, it may be concluded that, without Dopplers, the road identification is more ambiguous and less robust than with Dopplers.

Considering Figure 4, it should be noticed that the hypothesis never reaches the end of a carriageway. Two phenomena

\begin{tabular}{|c|c|c||c|c||c|c|c|}
\hline & \multicolumn{2}{|c|}{ One hypothesis } & \multicolumn{2}{c||}{ Several hypotheses } & \multicolumn{3}{c|}{ Total } \\
& OK & NOK & Amb. & NOK & OK & Amb. & NOK \\
\hline Mean & $97 \%$ & $3 \%$ & $98 \%$ & $2 \%$ & $89 \%$ & $8 \%$ & $3 \%$ \\
Best & $99 \%$ & $1 \%$ & $100 \%$ & $0 \%$ & $90 \%$ & $9 \%$ & $1 \%$ \\
Worst & $93 \%$ & $7 \%$ & $96 \%$ & $4 \%$ & $87 \%$ & $7 \%$ & $6 \%$ \\
\hline
\end{tabular}

Table I: Road Identification in open-sky conditions.
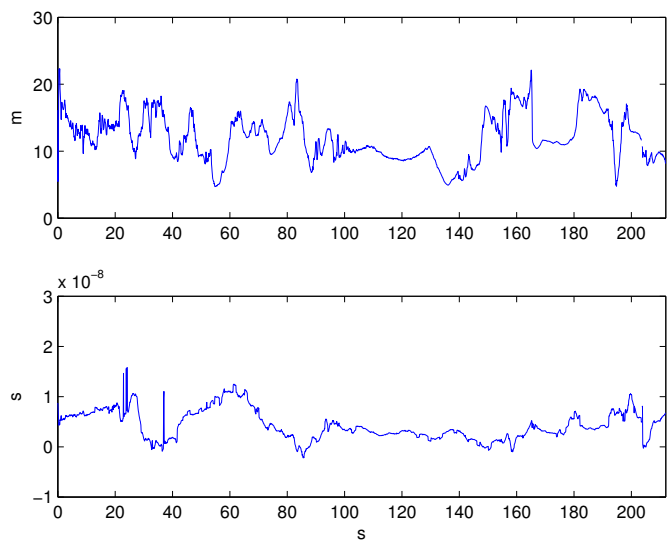

Figure 7: Estimation results with a good visibility: Position $-u p-$ and clock offset - down-.
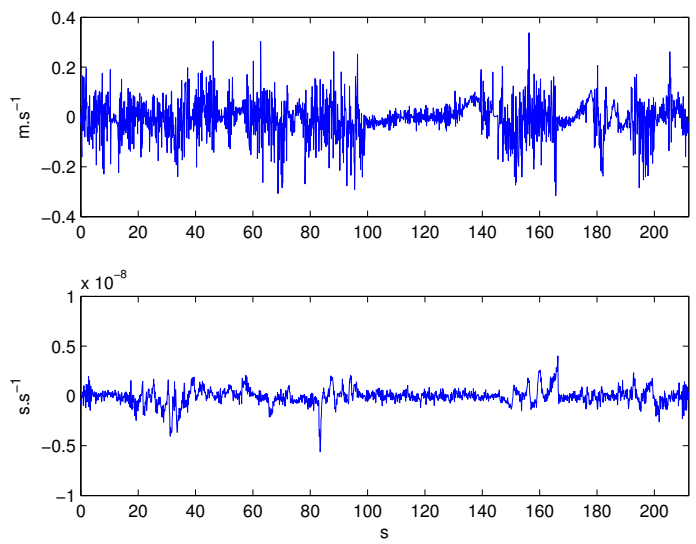

Figure 8: Estimation results with a good visibility: Velocity $-u p-$ and clock drift - down-.

can explain this behavior: when switching from one road to another, particles are unlikely to have a null abscissa, as a result of the model noise and the subtraction of the length of the previous carriageway. Moreover, the use of a Gaussian mixture on particle subset magnifies this phenomenon as shown in Figure 6. In this example, there are 4 Gaussians. When the Gaussians are distributed along two different roads, the mean values of all hypotheses are at some distance from the node of the junction.

\section{Performance under normal visibility conditions}

1) Road identification: The performance of the proposed method is here evaluated with a good satellite visibility (up to 9 satellites in this experiment). As the solver acts like a Monte Carlo method, the data set was processed one hundred times. Because of the limited accuracy of the manually-performed 
road selection ground truth, results were rounded to the nearest percent. Table I shows these figures where:

- OK means "Road identification correctly done (the correct road is the only hypothesis)".

- Amb. means "An ambiguous area is identified and the set of hypotheses includes the correct road".

- NOK means "Wrong selection (the correct ID is not included in the set of hypotheses)".

In spite of the map offset, the method delivers satisfactory results, since the wrong selection rate remains low. Moreover, ambiguous areas are well identified, and the best and worst cases have similar rates. These results show that the proposed method provides a good identification of ambiguous areas, a correct road identification and a low rate of wrong matches, despite the low map accuracy.

2) Positioning quality: Road identification is only one aspect of the problem. The quality of the position tracking also has to be evaluated. We consider here the mean estimate of the particle set. Figure 7 shows the estimation errors for the position and the receiver clock offset with respect to those computed by the receiver itself (these values are called "first order parameters"). Additionally, Figure 8 displays the estimation errors for the derivative parameters: velocity and clock drift.

Considering the first order parameters, the estimation errors are not centered because of the tight integration of the map in the fusion process. The mean value for the position error is between $12 \mathrm{~m}$ and $15 \mathrm{~m}$. This bias corresponds to the map offset. Identically, the clock offset estimation error is not centered, meaning that part of the map offset carries over to the clock offset estimation. Conversely, estimation errors for the derivative parameters are centered: the map constraint has a limited impact on these parameters. These results show that the proposed method provides a good estimation of location in open-sky conditions.

\section{E. Performance with few visible satellites}

In these experiments, urban canyons are simulated. Several satellites are simply removed from the computation using a sectoral mask (see Figure 9).

1) 3 available satellites: Table II shows the results of the road identification behavior. Regarding road identification, no significant variation is observed with respect to the full visibility condition, except for the worst case. Here, the wrong selection rate significantly increases. As the number of measurements is reduced, the effectiveness of road identification decreases. Despite the lack of measurement redundancy, the method still provides interesting results since the dispersion is limited.

2) 2 available satellites: Measuring only 2 satellites is a very challenging situation. In this case, the identification performance significantly decreases, as shown in Table III. Moreover, the dispersion of the results is significantly increased: the worst case gives about $50 \%$ wrong selections, whereas the best case has similar results to open-sky conditions. These results are due to the lack of redundancy with only 2 satellites. With 2 satellites only 5 measurements are available to estimate

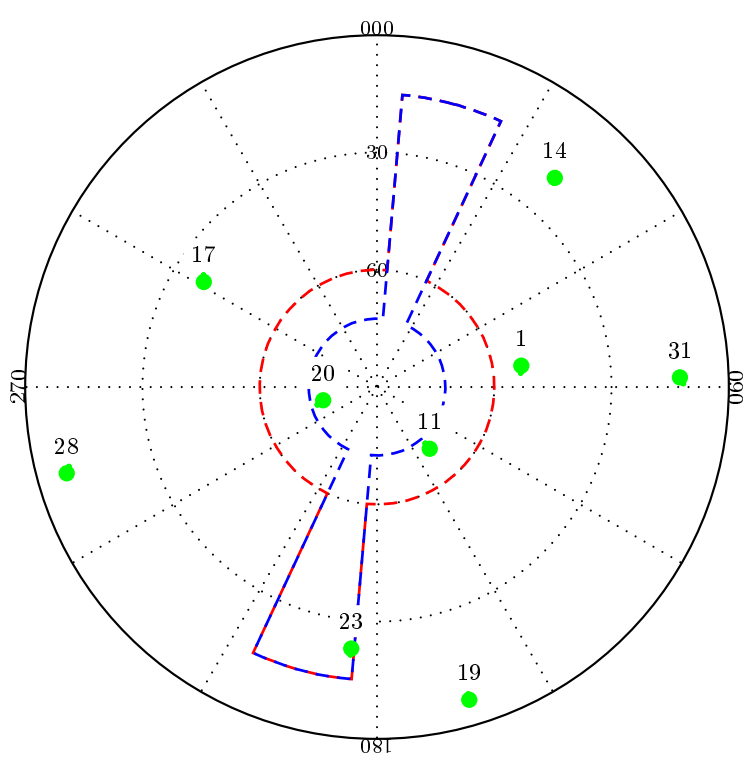

Figure 9: Sectoral masks used to simulate urban canyoning. Red line stands for the $3 \mathrm{SV}$ configuration and blue line for the $2 \mathrm{SV}$ configuration.

\begin{tabular}{|c|c|c||c|c||c|c|c|}
\hline & \multicolumn{2}{|c||}{ One hypothesis } & \multicolumn{2}{c||}{ Several hypotheses } & \multicolumn{3}{c|}{ Total } \\
& OK & NOK & Amb. & NOK & OK & Amb. & NOK \\
\hline Mean & $95 \%$ & $5 \%$ & $97 \%$ & $3 \%$ & $84 \%$ & $12 \%$ & $4 \%$ \\
Best & $99 \%$ & $1 \%$ & $99 \%$ & $1 \%$ & $87 \%$ & $12 \%$ & $1 \%$ \\
Worst & $88 \%$ & $12 \%$ & $94 \%$ & $8 \%$ & $77 \%$ & $12 \%$ & $11 \%$ \\
\hline
\end{tabular}

Table II: Road identification with 3 satellites.

\begin{tabular}{|c|c|c||c|c||c|c|c|}
\hline & \multicolumn{2}{|c|}{ One hypothesis } & \multicolumn{2}{c||}{ Several hypotheses } & \multicolumn{3}{c|}{ Total } \\
& OK & NOK & Amb. & NOK & OK & Amb. & NOK \\
\hline Mean & $83 \%$ & $17 \%$ & $86 \%$ & $14 \%$ & $71 \%$ & $13 \%$ & $16 \%$ \\
Best & $97 \%$ & $3 \%$ & $95 \%$ & $5 \%$ & $84 \%$ & $12 \%$ & $4 \%$ \\
Worst & $53 \%$ & $47 \%$ & $43 \%$ & $57 \%$ & $44 \%$ & $7 \%$ & $49 \%$ \\
\hline
\end{tabular}

Table III: Road identification with 2 satellites.

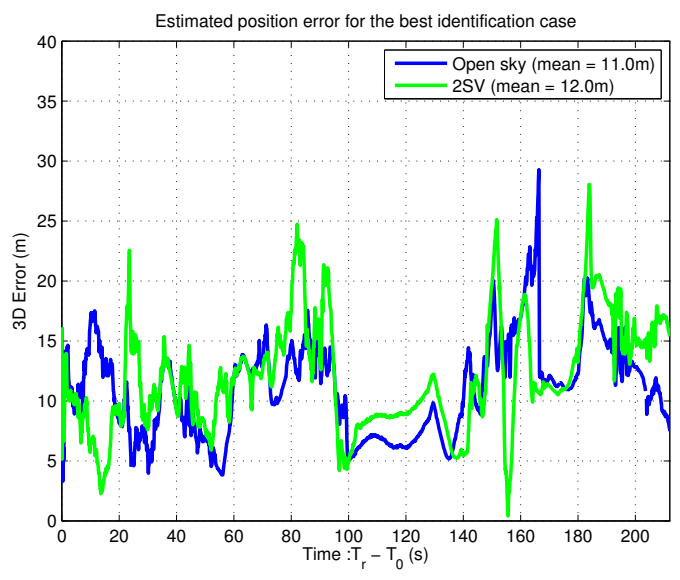

Figure 10: Positioning error with 2 satellites in the best case. Open sky conditions are also reported for comparison 

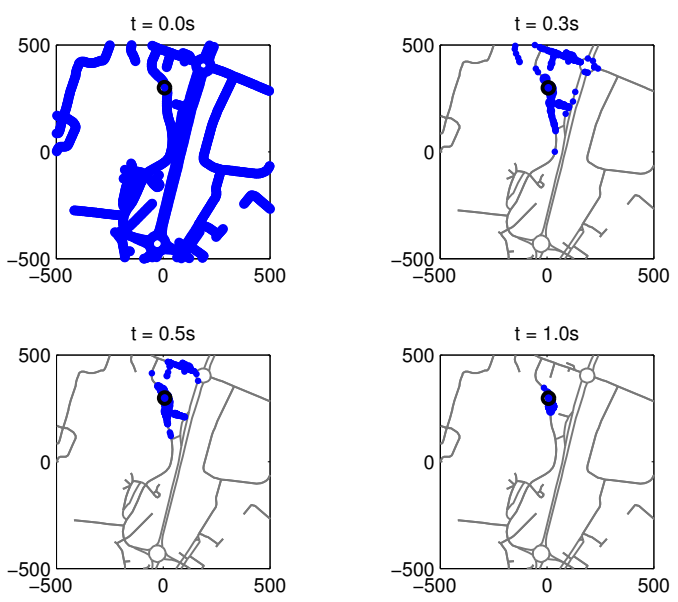

(a) Evolution of the particle set. The black circle is the ground truth.
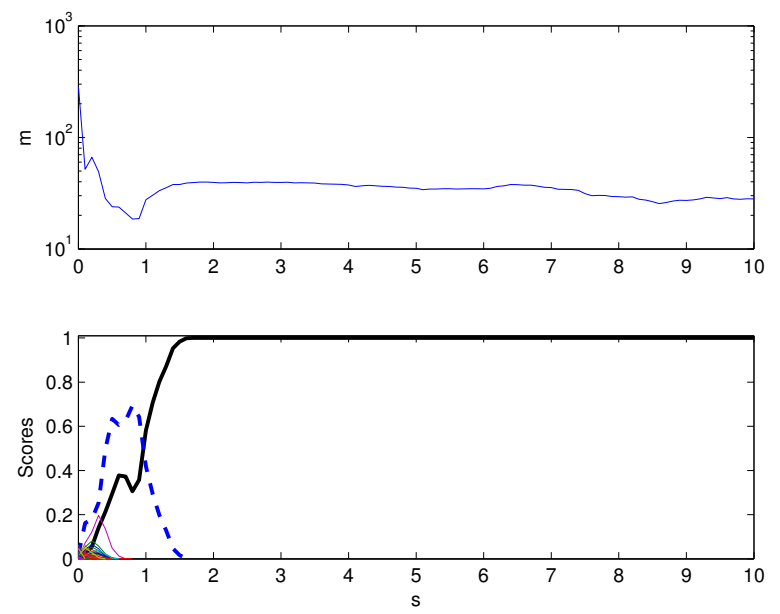

(b) Position error in meters (log scale) - Up- Hypotheses likelihoods -Down.

Figure 11: Initialization process for a moving vehicle.

the state vector, the dimension of which is 5. Owing to the absence of redundancy, an erroneous hypothesis can be more likely than the true one. Then, at a road junction, the particle set may be resampled according to this erroneous hypothesis, leading to a filter degeneracy.

Figure 10 shows the positioning errors obtained in the best case of road identification. With different satellite configurations, errors are quite similar. This is a important result: the method is able to track the vehicle location with only two measurements when road identification is correctly solved. In practice, if the road identification is correctly done, one can rely on the computed position between two junctions even with only 2 satellites.

Thus, one may conclude that information redundancy is vital for performing road identification efficiently, but fewer measurements are needed for position tracking.

\section{F. Global initialization evaluation}

Let now look at the behavior of the global initialization strategy, as proposed in Section IV-D. Considering the good
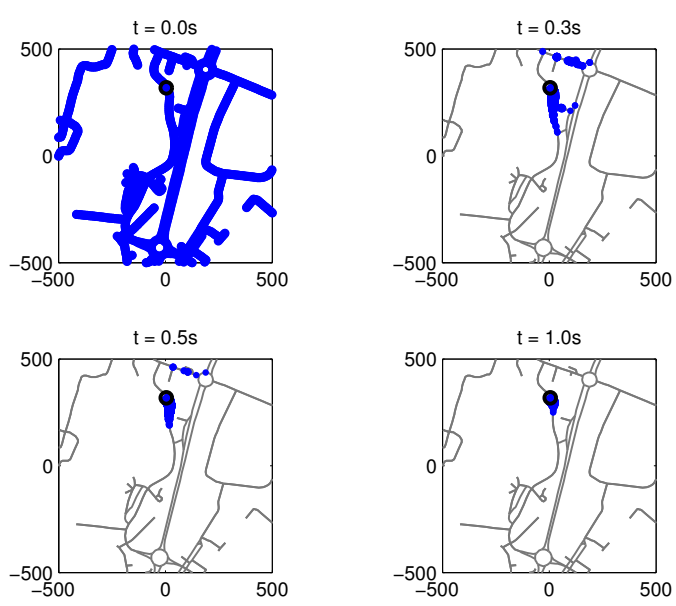

(a) Evolution of the particle set.The black circle is the ground truth.
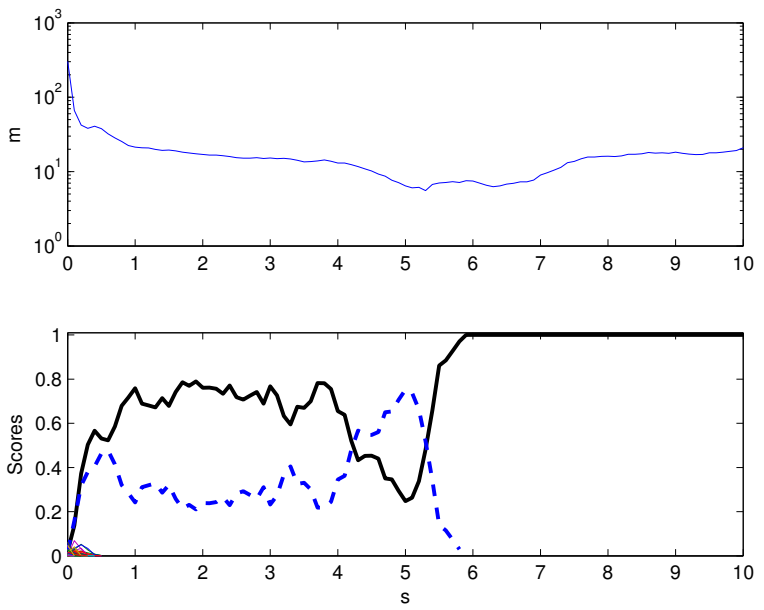

(b) Position error in meters (log scale) - Up- Hypotheses likelihoods -Down.

Figure 12: Initialization process for a stopped vehicle. The vehicle starts moving at $t=4 s$.

performance of the map-matching tracking in open-sky conditions, only limited visibility conditions are investigated here using 3 satellites (See Figure 9 for the satellite locations). The convergence of the method is considered for a moving vehicle (Figure 11) and a stationary vehicle (Figure 12). For each test case, sub-figure (a) presents the convergence of the particle set during the first second. In both cases, convergence is reached in approximately 1 second and a positioning error of $\sim 12 \mathrm{~m}$ is obtained (which is consistent with the observed map offset). This fast convergence means a fast reduction of the particle set size, which is an interesting feature for an embedded system.

In order to assess the initialization process, positioning and road identification are to be considered jointly. Subfigures (b) of Figures 11 and 12 show the evolution of those parameters. The upper curve provides the positioning error for the barycenter of the particle set (the error is plotted in a semi$\log$ ) and the lower curve depicts the evolution of the hypothesis scores. The two carriageways forming part of the correct road are highlighted: the solid line represents the correct driving 
direction and the dashed line represents the opposite direction.

The proposed initialization process gives interesting results. When the vehicle is moving, the convergence is fast and correct. When the vehicle is motionless, the algorithm converges toward a bi-modal solution corresponding to the same carriageway. This is correct and it is the expected behavior. Indeed, the direction of the vehicle is not observable in this case. The estimation process can only rely on the position of the vehicle. When the vehicle starts moving, the road identification is correctly performed in a few steps with a time constant similar to the previous case. This is an interesting result compared to a standard GPS receiver, which would be unable to provide a position solution in such a situation with less than 4 satellites.

\section{CONCLUSION}

In this paper, a novel approach to map-matching has been proposed which does not require knowledge of a precise global position. This approach relies on a Bayesian formalism well adapted to hybrid state estimation, in which discrete and continuous components are to be estimated simultaneously. Moreover, this approach is also able to handle multihypothesis tracking, which is an interesting characteristic for initialization and tracking in ambiguous areas. The state space is described as a Jump Markov System, given the assumption of map-constrained motion. Using a factored form of the positioning posterior, a set of constrained hypotheses can be estimated with a limited number of hypotheses.

This general modeling has been applied to the problem of matching pseudoranges and Doppler GPS measurements to standard navigable maps. Topology and geometry are both used. The network topology provides the support for the Markov chain and network geometry depicts the possible motion of every hypothesis. The factoring of the problem can be harnessed through the use of a MPF. Moreover, an adaptive re-sampling strategy allows the particle set to be resized in relation to the estimation complexity. An initialization scheme has been also presented to estimate the global position with little prior knowledge about the position, by assuming only that the road cache contains the solution.

Experimental results have shown that such an approach is valid. Moreover, the use of GPS Doppler measurements is very fruitful and improves the map-matching significantly. The method is robust to map offsets. It achieves a good road identification rate and a good positioning precision even if only 3 GPS satellites are available. With only 2 satellites in view, the performance of the road identification decreases significantly. Nevertheless, a good positioning can still be achieved, which is an interesting property when navigating in dense areas where GPS outages frequently occur. Furthermore, the proposed initialization process is able to converge in terms of road identification and positioning with only 3 satellites. This interesting feature can also be used to initialize a positioning system in cases of limited satellite visibility. Moreover, since the required number of particles is reasonable, this approach should be easily embedded in small devices.

Further improvements will consider the integrity monitoring of the positioning hypotheses using the same framework, since this information is of great interest for many ITS applications. In urban areas, GPS signal often suffers from multipath and attenuation leading to erroneous measurements. This should be taken into consideration for further improvement as this framework may enhance the detection of faulty measurements.

\section{REFERENCES}

[1] F. Abdallah, G. Nassreddine, and T. Denoeux. A multiple-hypothesis map matching method suitable for weighted and box-shaped state estimation for localization. IEEE Trans. Intell. Transp. Syst., 12(4):14951510, December 2011.

[2] C. Andrieu, M. Davy, and A. Doucet. Efficient particle filtering for jump markov systems - applications to time-varying autoregressions. IEEE Trans. Signal Processing, 51(7):1762-1770, 2003.

[3] Ph. Bonnifait, M. Jabbour, and G. Dherbomez. Real-time implementation of a gis-based localization system for intelligent vehicles. EURASIP Journal on Embedded Systems, pages 12-24, June 2007.

[4] Ph. Bonnifait, J. Laneurit, and C. Fouque. Multi-hypothesis mapmatching using particle filtering. In ITS World Congress, Stockholm, Sept. 2009.

[5] Y.J. Cui and S.S. Ge. Autonomous vehicle positioning with gps in urban canyon environments. IEEE Trans. on Rob. and Automation, 19:15-25, 2003.

[6] N. de Freitas, R. Dearden, F. Hutter, R. Morales-Menendez, J. Mutch, and D. Poole. Diagnosis by a waiter and a mars explorer. In Proceedings of the IEEE, special issue on Sequential State Estimation, volume 92, pages $455-468,2003$.

[7] M. El Badaoui El Najjar and Ph. Bonnifait. Road selection using multi-criteria fusion for the road-matching problem. IEEE Trans. Intell. Transp. Syst., 8:279-291, June 2007.

[8] C. Fouque and Ph. Bonnifait. Tightly coupled GIS data in GNSS fix computation with integrity test. International Journal of Intelligent Information and Database Systems, 2(2):167-186, 2008.

[9] D. Fox. Kld-sampling: Adaptive particle filters. In MIT Press, editor, In Advances in Neural Information Processing Systems, volume 14, pages 713-720, 2001.

[10] J. S. Greenfeld. Matching gps observation to locations on a digital map. Proceedings of th 81st Annual Meeting of the Transportation Research Board, 2002.

[11] G. Grisetti, C. Stachniss, and W. Burgard. Improved techniques for grid mapping with rao-blackwellized particle filters. Robotics, IEEE Transactions on, 23(1):34 -46, feb. 2007.

[12] F. Gustafsson, F. Gunnarsson, N Bergman, U Forssell, J. Jansson, R. Karlsson, and P.J. Nordlund. Particle filters for positioning, navigation, and tracking. IEEE Trans on Signal Processing, 50(2):425-437, Feb. 2002.

[13] M. Jabbour, Ph. Bonnifait, and V. Cherfaoui. Map-Matching Integrity using Multi-Sensor Fusion and Multi-Hypothesis Road Tracking. Journal of Intelligent Transportation Systems, 12(4):189-201, 102008.

[14] A. Lahrech, C. Boucher, and J-C. Noyer. Accurate vehicle positioning in urban areas. IECON Proceedings (Industrial Electronics Conference), 5:486-490, 2005

[15] J. Laneurit, R. Chapuis, and Chausse F. Accurate vehicle positioning on a numerical map. Int. J. of Control, Automation and System, 3(1):15-31, 2005.

[16] O. Mazhelis. Using recursive bayesian estimation for matching gps measurements to imperfect road network data. In IEEE International Conference on Intelligent Transportation Systems, pages 1492-1497, Madeira Island, Portugal, September 19-22 2010.

[17] M.A. Quddus, W.Y. Ochieng, and R.B. Noland. Current map-matching algorithms for transport applications: State-of-the art and future research directions. Transportation Research Part C: Emerging Technologies, 15(5):312-328, 2007

[18] A. Rae and O. Basir. Improving vehicle positioning and visual feature estimates through mutual constraint. In IEEE Intelligent Transportation Systems Conference, pages 778 -783, 30 2007-oct. 32007.

[19] B. Ristic, S. Arulampalam, and N. Gordon. Beyond the Kalman Filter Particle Filters for Tracking Applications. Artech House, 2004.

[20] N. Samama. Global Positionning: Technologies and Performance. Wiley-Interscience, 2008.

[21] T. Schön, T. Gustafsson, and P.J. Nordlund. Marginalized Particle Filters for Mixed Linear Nonlinear State-Space Models. IEEE Trans. on Signal Processing, 53:2279-2289, 2005. 
[22] I. Skog and P. Handel. In-car positioning and navigation technologies;a survey. IEEE Trans. Intell. Transp. Syst., 10(1):4 -21, March 2009.

[23] C. Smaili, M. El Badaoui El Najjar, and C. Charpillet. A road matching method for precise vehicle localization using hybrid bayesian network. Journal of Intelligent Transportation Systems, 12(4), October 2008.

[24] S. Syed. Gps-based map matching in the pseudorange measurement domain. In Proc. of GNSS 2004, Long Beach, CA, 2004.

[25] G.E. Taylor and G. Blewitt. Intelligent Positioning: GIS-GPS Unification. John Wiley and Sons Ltd., London, UK, 2006.

[26] S. Thrun, W. Burgard, and D. Fox. Probabilistic Robotics. The MIT Press, 2005.

[27] R. Toledo-Moreo, D. Bétaille, and F. Peyret. Lane-level integrity provision for navigation and map matching with GNSS, dead reckoning and enhanced maps. IEEE Trans. Intell. Transp. Syst., 11(1):100-112, March 2010.

[28] C.E. White, D. Bernstein, and A.L. Kornhauser. Some map matching algorithms for personal navigation assistants. Transportation Research Part C: Emerging Technologies, 8(1-6):91-108, 2000.

[29] J. Zhang, K. Zhang, R. Grenfell, and R. Deakin. Gps satellite velocity and acceleration determination using the broadcast ephemeris. Journal of Navigation, 59(2):293-305, 2006.

\section{APPENDIX}

Let detail the components of the Jacobian matrix relative to the constrained GPS measurements. For a particular satellite, a PR and a Doppler measurements provide two rows in $G_{k}^{\mathcal{I}}$ :

$$
G_{k}^{\mathcal{I}}=\left[\begin{array}{cccc}
\frac{\partial \rho}{\partial l} & 0 & 1 & 0 \\
\frac{\partial \dot{\rho}}{\partial l} & \frac{\partial \dot{\rho}}{\partial v} & 0 & 1
\end{array}\right]
$$

Next, we focus on the expression of the component relative to the velocity and abscissa of the hypothesis.

\section{A. Jacobian matrix for the constrained pseudorange}

The constrained pseudorange model is:

$$
\rho_{k}=\left\|X_{k}^{\mathcal{I}}-X_{k}^{s}\right\|+d_{k}
$$

Let consider the term relative to the curvilinear abscissa $l$ :

$$
\frac{\partial \rho}{\partial l}=\frac{1}{2 \cdot\left\|X_{k}^{\mathcal{I}}-X_{k}^{s}\right\|} \cdot \frac{\partial}{\partial l}\left(\left(X_{k}^{\mathcal{I}}-X_{k}^{s}\right)^{2}\right)
$$

Since $X_{k}^{s}$ does not depend on the curvilinear abscissa:

$$
\frac{\partial}{\partial l}\left(\left(X_{k}^{\mathcal{I}}-X_{k}^{s}\right)^{2}\right)=2 \cdot\left(X_{k}^{\mathcal{I}}-X_{k}^{s}\right) \bullet \frac{\partial X_{k}^{\mathcal{I}}}{\partial l}
$$

Considering Eq.(26), the derivative of $X_{k}^{\mathcal{I}}$ is given by:

$$
\frac{\partial X_{k}^{\mathcal{I}}}{\partial l}=U_{i}^{\mathcal{I}}
$$

Thus, the corresponding term of the Jacobian matrix is given by the dot product, denoted $\bullet$, of the segment direction and the line-of-sight vector $u_{\mathrm{los}}$ :

$$
\frac{\partial \rho}{\partial l}=u_{\operatorname{los}} \bullet U_{i}^{\mathcal{I}}
$$

\section{B. Jacobian matrix for the constrained Doppler}

The constrained Doppler model is given by:

$$
\dot{\rho}_{k}=\left(V_{k}^{\mathcal{I}}-V_{k}^{s}\right) \bullet u_{\mathrm{los}}+\dot{d}_{k}
$$

First, we consider the Jacobian matrix component relative to the hypothesis speed $v$ :

$$
\frac{\partial \dot{\rho}}{\partial v}=\frac{\partial V_{k}^{\mathcal{I}}}{\partial v} \bullet u_{\operatorname{los}}
$$

The corresponding component is also given by the dot product of the segment direction and the line-of-sight vector:

$$
\frac{\partial \dot{\rho}}{\partial v}=U_{i}^{\mathcal{I}} \bullet u_{\operatorname{los}}
$$

The component relative to the hypothesis abscissa is:

$$
\frac{\partial \dot{\rho}}{\partial l}=\left(V_{k}^{\mathcal{I}}-V_{k}^{s}\right) \bullet \frac{\partial u_{\operatorname{los}}}{\partial l}
$$

The variation of the line-of-sight vector is given by:

$$
\frac{\partial u_{\operatorname{los}}}{\partial l}=\frac{1}{\left\|X_{k}^{\mathcal{I}}-X_{k}^{s}\right\|^{2}} \cdot\left[\left\|X_{k}^{\mathcal{I}}-X_{k}^{s}\right\|-\frac{\left(X_{k}^{\mathcal{I}}-X_{k}^{s}\right)^{2}}{\left\|X_{k}^{\mathcal{I}}-X_{k}^{s}\right\|^{3}}\right] \cdot \frac{\partial X_{k}^{\mathcal{I}}}{\partial l}
$$

Thus, the corresponding term is:

$$
\frac{\partial \dot{\rho}}{\partial l}=\left[u_{\operatorname{los}} \times\left(u_{\operatorname{los}} \times \frac{V_{k}^{\mathcal{I}}-V_{k}^{s}}{\left\|X_{k}^{\mathcal{I}}-X_{k}^{s}\right\|}\right)\right] \bullet U_{i}^{\mathcal{I}}
$$

where $\times$ stands for the cross product.

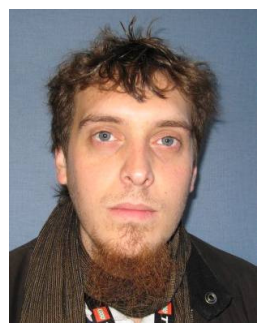

Clément Fouque was born in Nogent-sur-Marne, France, in 1982. He received a Master Degree in Mechanical and System Engineering from the Pierre et Marie Curie University (UPMC), Paris, France in 2006 and Ph.D. degree in Computer Science from the University of Technologie of Compiègne (UTC), Compiègne, France, in September 2010. Since November 2010, he is a post-doctoral research associate with Institut Pascal (formerly LASMEA CNRS 6602), Clermont-Ferrand, France. His current research interest includes sensor fusion and autonomous transportation systems.

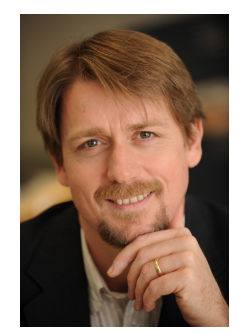

Philippe Bonnifait graduated from the Ecole Superieure d'Electronique de l'Ouest, France, in 1992 and received the Ph.D. degree in automatic control and computer science from the Ecole Centrale de Nantes, France, in 1997. In December 2005, he obtained the Habilitation à Diriger des Recherches from the University of Technology of Compiègne (UTC). He joined the Institut de Recherche en Communications et Cybernétique de Nantes (IRCCyN UMR 6597), France, in 1993. Since September 1998, he is with HEUDIASYC CNRS laboratory, France. He was Associate Professor at UTC, computer science department, from Sept. 1998 to Aug. 2007. Since Sept. 2007, he is Professor and head of a research group on Robotics, Automation and Embedded Systems. His current research interests are in Intelligent Vehicles and Advanced Driving Assistance Systems, with particular emphasis on dynamic ego-localisation based on multisensor-fusion and tracking (GNSS, dead-reckoning and GIS). 